\title{
Isovector and isoscalar tensor form factors of $N(1535) \rightarrow N$ transition in light-cone QCD
}

\author{
Ulaş Özdem(1)* \\ Health Services Vocational School of Higher Education, Istanbul Aydin University, \\ Sefakoy-Kucukcekmece, 34295 Istanbul, Turkey
}

(Received 25 April 2020; accepted 19 June 2020; published 1 July 2020)

\begin{abstract}
We have applied isovector and isoscalar tensor current to evaluate the tensor form factors of the $N(1535) \rightarrow N$ transition with the help of the light-cone QCD sum rule method. In numerical computations, have used the most general forms of the interpolating current for the nucleon and the tensor current together with two different sets of the input parameters in the distribution amplitudes (DAs) of the $N(1535)$ state. We have obtained that the values of $N(1535) \rightarrow N$ transition tensor form factors are very sensitive to the input parameters of the DAs of the $N(1535)$ state. We have acquired that the $Q^{2}$ dependence of $N(1535) \rightarrow$ transition tensor form factors is well defined by a $p$-pole fit function.
\end{abstract}

DOI: 10.1103/PhysRevD.102.014001

\section{INTRODUCTION}

The essential subject of QCD is to understand the internal structure of hadrons and their features in terms of degrees of freedom of quark gluons. Hadron charges described as matrix elements of tensor, axial, and vector currents between hadron states include complete knowledge about the internal structure of the hadron. At the twisttwo level, the corresponding charges are characterized by the helicity distribution $g_{1}(x)$, transversity distribution $h_{1}(x)$, and unpolarized distribution $f_{1}(x)$ function of the quark. More generally, at the leading twist, eight generalized parton distributions (GPDs) encompass full knowledge on the internal structure of hadrons: four chiral-odd spin-dependent GPDs $H_{T}(x, \xi, t), E_{T}(x, \xi, t), \tilde{H}_{T}(x, \xi, t)$, and $\tilde{E}_{T}(x, \xi, t)$; two chiral-even spin-dependent GPDs $\tilde{H}(x, \xi, t)$ and $\tilde{E}(x, \xi, t)$; and two chiral-even spinindependent GPDs $H(x, \xi, t)$ and $E(x, \xi, t)$, where $\xi$ is the skewness and $t=-Q^{2}$ is the squared momentum transfer [1-4]. These observables include important knowledge about the internal structure of the hadron. They characterize, e.g., how partons are distributed in the transverse plane according to motion of the hadron or the contribution of quark orbital angular momentum to total angular momentum of the hadron. The helicity and unpolarized distribution functions can be extracted from inclusive deep-inelastic scattering data because of their

\footnotetext{
*ulasozdem@aydin.edu.tr
}

Published by the American Physical Society under the terms of the Creative Commons Attribution 4.0 International license. Further distribution of this work must maintain attribution to the author(s) and the published article's title, journal citation, and DOI. Funded by SCOAP. chiral-even nature. In the forward limit, they are related to the electromagnetic, axial, and pseudoscalar form factors [5]. However, the transversity distribution function, which is related to the tensor form factors in the forward limit, has chiral-odd nature, so there is a big experimental problem to measure it. It can be acquired Drell-Yan processes and semi-inclusive deep inelastic scattering, as distributions of transversity do appear at leading twist in the cross section. Photo- and electroproduction of mesons off the polarized nucleons and the transversely polarized DrellYan process are recommended as suitable ways to measure transversity distribution. In Ref. [6], transversity distribution of the nucleon was extracted using the experimental data from the COMPASS [7], HERMES [8], and Belle [9] Collaborations. Afterward, in Ref. [10], the tensor charge of the nucleon was extracted in the framework of the covariant quark-diquark model. Moreover, tensor form factors of the nucleon have been investigated by the help of QCD sum rule [11,12], axial vector meson dominance model [13], quark model [14,15], chiral quark soliton model [16,17], light-cone QCD sum rule [18,19], dihadron production [20], lattice QCD [21-23], relativistic confined quark model [24], and Skyrme model [25]. Besides, the tensor form factors of the octet hyperons are investigated in the framework of the chiral quark soliton model [17] and light-cone QCD sum rule [26].

Form factors play a crucial role in our comprehension of the tomography of baryons. The tensor form factors are missing part of this tomography. Recently, the measurements of exclusive electroproduction of pseudoscalar mesons ( $\pi$ and $\eta$ mesons) has demonstrated that these processes are responsive to chiral-odd GPDs [27-30]. Photo- and electroproduction of pseudoscalar mesons can be used to extract the tensor form factors of baryons 
[27]. In the short run, remarkably more accurate measurements of the nucleon tensor form factors are expected at Jefferson Laboratory (JLab) by the CLAS Collaboration. Besides, the experiments designed at CLAS Collaboration have been aimed to investigate features of electroexcitation of nucleon resonances in photo- and electroproduction reactions [31]. Inspired by the future experiments at JLab, we aim to investigate the isovector and isoscalar tensor form factor $N(1535) \rightarrow N$ transition up to a momentum transfer of $Q^{2} \leq 10 \mathrm{GeV}^{2}$ with the help of the light-cone QCD sum rule. To our knowledge, this is the first study in the literature committed to the examination of the $N(1535) \rightarrow N$ transition tensor form factors. In the lightcone QCD sum rule method, the hadronic observables are described in connection with the properties of the vacuum and distribution amplitudes (DAs) of the hadrons under investigation [32-34]. Since the hadronic observables are described in connection with the features of the QCD vacuum and the DAs, any ambiguity in these variables reflects the ambiguity of the predictions of the hadronic observables. Note that the electromagnetic [35], axial [36], and gravitational [37] form factors for $N(1535) \rightarrow N$ transition have been evaluated with the help of light-cone QCD sum rule.

This article is organized in the following manner: In Sec. II, we present the details of our light-cone QCD sum rule calculations. In Sec. III, we analyze the obtained results and give our conclusions.

\section{ISOVECTOR AND ISOSCALAR TENSOR FORM FACTORS OF $N(1535) \rightarrow N$ TRANSITION}

The matrix element of the isovector and isoscalar tensor current between nucleon and $N(1535)$ baryons is defined by three dimensionless invariant form factors as presented $[38,39]$ :

$$
\begin{aligned}
& \left\langle N\left(p^{\prime}\right)\left|J_{\mu \nu}\right| N(1535)(p)\right\rangle \\
& =\bar{u}\left(p^{\prime}\right)\left[i \sigma_{\mu \nu} H_{T}^{I=0,1}\left(Q^{2}\right)+\frac{\gamma_{\mu} q_{\nu}-\gamma_{\nu} q_{\mu}}{2 \bar{m}} E_{T}^{I=0,1}\left(Q^{2}\right)\right. \\
& \left.\quad+\frac{\tilde{P}_{\mu} q_{\nu}-\tilde{P}_{\nu} q_{\mu}}{2 \bar{m}^{2}} \tilde{H}_{T}^{I=0,1}\left(Q^{2}\right)\right] \gamma_{5} u(p),
\end{aligned}
$$

where $\quad \bar{m}=\left(m_{N}+m_{N(1535)}\right) / 2, \quad \sigma_{\mu \nu}=\frac{i}{2}\left[\gamma_{\mu}, \gamma_{\nu}\right], \quad q=$ $p-p^{\prime}, \quad \tilde{P}=p^{\prime}+p$, and $F^{I=1}=F^{u}-F^{d}$ and $F^{I=0}=$ $F^{u}+F^{d}$ for any of the form factors, $F=E_{T}, H_{T}$, or $\tilde{H}_{T}$.

To derive the light-cone QCD sum rules for isovector and isoscalar tensor form factors of $N(1535) \rightarrow N$ transition, we consider the subsequent correlator for our analysis:

$$
\Pi_{\mu \nu}(p, q)=i \int d^{4} x e^{i q x}\left\langle 0\left|\mathcal{T}\left[J_{N}(0) J_{\mu \nu}(x)\right]\right| N(1535)(p)\right\rangle,
$$

where $J_{\mu \nu}(x)$ is the tensor current and $J_{N}(0)$ are interpolating currents for nucleon states. The explicit forms of $J_{N}(0)$ and $J_{\mu \nu}(x)$ are given as

$$
\begin{aligned}
& J_{N}(0)=2 \epsilon^{a b c} \sum_{\ell=1}^{2}\left(u^{a T}(x) C J_{1}^{\ell} u^{b}(x)\right) J_{2}^{\ell} d^{c}(x), \\
& J_{\mu \nu}(x)=\bar{u}^{d}(x) i \sigma_{\mu \nu} u^{d}(x) \pm \bar{d}^{e}(x) i \sigma_{\mu \nu} d^{e}(x),
\end{aligned}
$$

respectively, where $J_{1}^{1}=I, J_{1}^{2}=J_{2}^{1}=\gamma_{5}$, and $J_{2}^{2}=t$, which is an arbitrary parameter that fixes the mixing of two local operators, and $C$ denotes charge conjugation.

To acquire the sum rules for isovector and isoscalar tensor form factors of the $N(1535) \rightarrow N$ transition, the correlator in Eq. (2) is obtained from the subsequent three steps:

(i) The correlator is saturated by a complete set of hadronic states, which have the same quantum numbers as interpolating currents (hadronic representation).

(ii) The correlator is obtained in connection with quark and gluon degrees of freedom interacting with nonperturbative QCD vacuum (QCD representation).

(iii) Then match these two independent representations of the correlator to one another employing the quarkhadron duality ansatz. To keep under control undesirable contributions coming from the higher and excited states, we perform a Borel transformation, in addition to continuum subtraction to both representations of the obtained corresponding sum rules.

As we mentioned above, in order to evaluate the correlator in connection with hadron features, a complete hadronic set with the same quantum numbers as the interpolation currents is inserted. After that, the correlation function becomes

$$
\begin{aligned}
\Pi_{\mu \nu}^{\mathrm{Had}}(p, q)= & \sum_{s^{\prime}} \frac{\left\langle 0\left|J_{N}(0)\right| N\left(p^{\prime}, s^{\prime}\right)\right\rangle}{m_{N}^{2}-p^{\prime 2}} \\
& \times\left\langle N\left(p^{\prime}, s^{\prime}\right)\left|J_{\mu \nu}(x)\right| N(1535)(p, s)\right\rangle+\cdots,
\end{aligned}
$$

where

$$
\left\langle 0\left|J_{N}(0)\right| N\left(p^{\prime}, s^{\prime}\right)\right\rangle=\lambda_{N} u_{N}\left(p^{\prime}, s^{\prime}\right),
$$

with $\lambda_{N}$ and $u_{N}\left(p^{\prime}, s^{\prime}\right)$ being the residue and Dirac spinor of nucleon, respectively. Summation over the spins of the nucleon is performed as

$$
\sum_{s^{\prime}} u_{N}\left(p^{\prime}, s^{\prime}\right) \bar{u}_{N}\left(p^{\prime}, s^{\prime}\right)=\not p^{\prime}+m_{N} .
$$

Substituting Eqs. (1), (5), and (6) into Eq. (4), we acquire the correlator in the way of the hadronic features as 
$\Pi_{\mu \nu}^{\mathrm{Had}}(p, q)=\frac{\lambda_{N}}{m_{N}^{2}-p^{\prime 2}}\left(\not p^{\prime}+m_{N}\right)\left[i \sigma_{\mu \nu} H_{T}^{I=0,1}\left(Q^{2}\right)+\frac{\gamma_{\mu} q_{\nu}-\gamma_{\nu} q_{\mu}}{2 \bar{m}} E_{T}^{I=0,1}\left(Q^{2}\right)+\frac{\tilde{P}_{\mu} q_{\nu}-\tilde{P}_{\nu} q_{\mu}}{2 \bar{m}^{2}} \tilde{H}_{T}^{I=0,1}\left(Q^{2}\right)\right] \gamma_{5} u(p)$.

The next step is to evaluate the correlator in Eq. (2) with respect to quarks and gluon properties in the deep Euclidean region. Employing the expression for $J_{N}(0)$ and $J_{\mu \nu}(x)$ and Wick's theorem, the QCD representation of the correlator is obtained as

$$
\begin{aligned}
\Pi_{\mu \nu}^{\mathrm{QCD}}(p, q)= & -\int d^{4} x e^{i q x}\left[\left\{\left(\gamma_{5}\right)_{\gamma \delta} C_{\alpha \beta}\left(i \sigma_{\mu \nu}\right)_{\omega \rho}+t(I)_{\gamma \delta}\left(C \gamma_{5}\right)_{\alpha \beta}\left(i \sigma_{\mu \nu}\right)_{\omega \rho}\right\}\right. \\
& \times\left\{\left\langle 0\left|\epsilon^{a b c} u_{\sigma}^{a}(0) u_{\theta}^{b}(x) d_{\phi}^{c}(0)\right| N(1535)(p)\right\rangle\left(\delta_{\sigma}^{\alpha} \delta_{\theta}^{\rho} \delta_{\phi}^{\beta} S_{u}(-x)_{\delta \omega}+\delta_{\sigma}^{\delta} \delta_{\theta}^{\rho} \delta_{\phi}^{\beta} S_{u}(-x)_{\alpha \omega}\right)\right. \\
& \left.\left. \pm\left\langle 0\left|\epsilon^{a b c} u_{\sigma}^{a}(0) u_{\theta}^{b}(0) d_{\phi}^{c}(x)\right| N(1535)(p)\right\rangle \delta_{\sigma}^{\alpha} \delta_{\theta}^{\delta} \delta_{\phi}^{\rho} S_{d}(-x)_{\beta \omega}\right\}\right],
\end{aligned}
$$

where $S_{q}(x)$ is the light-quark propagator and it is given as $\left(m_{q}=0\right)$

$$
S_{q}(x)=i \frac{\not x}{2 \pi^{2} x^{4}}-\frac{\langle\bar{q} q\rangle}{12}-\frac{\langle\bar{q} \sigma \cdot G q\rangle}{192} x^{2}-\frac{i g_{s}}{32 \pi^{2} x^{2}} G^{\mu \nu}(x)\left[\not x \sigma_{\mu \nu}+\sigma_{\mu \nu} x\right] .
$$

The $\left\langle 0\left|\epsilon^{a b c} u_{\sigma}^{a}\left(x_{1}\right) u_{\theta}^{b}\left(x_{2}\right) d_{\phi}^{c}\left(x_{3}\right)\right| N(1535)(p)\right\rangle$ matrix element in Eq. (8) is can be written in terms of the DAs of the $N(1535)$ state, and it is necessary for further computations. The comprehensive expression of this matrix elements are presented in Ref. [40]. After employing the explicit forms of the above matrix elements and the light-quark propagator, we acquire expressions in $x$ space. Then we apply Fourier transforms to transfer these expressions into the momentum space.
The desired light-cone sum rules are obtained by matching both representations of the correlation function. In order to do this, we have to choose different and independent Lorentz structures. For this purpose, we choose $p_{\mu} q_{\nu} \gamma_{5}$, $p_{\mu} \gamma_{\nu} \gamma_{5}$, and $p_{\mu} q_{\nu} q \gamma$ structures for $E_{T}^{I=0,1}\left(Q^{2}\right), H_{T}^{I=0,1}\left(Q^{2}\right)$, and $\tilde{H}_{T}^{I=0,1}\left(Q^{2}\right)$ form factors, respectively. As a result, we get the light-cone sum rules

$$
\begin{array}{cc}
E_{T}^{I=1}\left(Q^{2}\right) \frac{\lambda_{N}}{m_{N}^{2}-p^{\prime 2}}=\bar{m} \Pi_{1}^{\mathrm{QCD}}, & E_{T}^{I=0}\left(Q^{2}\right) \frac{\lambda_{N}}{m_{N}^{2}-p^{\prime 2}}=\bar{m} \Pi_{2}^{\mathrm{QCD}}, \\
H_{T}^{I=1}\left(Q^{2}\right) \frac{\lambda_{N}}{m_{N}^{2}-p^{\prime 2}}=-\frac{1}{2} \Pi_{3}^{\mathrm{QCD}}, & H_{T}^{I=0}\left(Q^{2}\right) \frac{\lambda_{N}}{m_{N}^{2}-p^{\prime 2}}=-\frac{1}{2} \Pi_{4}^{\mathrm{QCD}}, \\
\tilde{H}_{T}^{I=1}\left(Q^{2}\right) \frac{\lambda_{N}}{m_{N}^{2}-p^{\prime 2}}=-\bar{m}^{2} \Pi_{5}^{\mathrm{QCD}}, & \tilde{H}_{T}^{I=0}\left(Q^{2}\right) \frac{\lambda_{N}}{m_{N}^{2}-p^{\prime 2}}=-\bar{m}^{2} \Pi_{6}^{\mathrm{QCD}} .
\end{array}
$$

The $\Pi_{i}^{\mathrm{QCD}}$ functions appearing in Eqs. (10)-(12) are quite long and not illuminating. However, as an example, we give the result of the $\Pi_{1}^{\mathrm{QCD}}$. The remaining five of these functions have more or less similar forms:

$$
\begin{aligned}
\Pi_{1}^{\mathrm{QCD}}= & 2 m_{N(1535)}^{2}\left\{\int _ { 0 } ^ { 1 } d \alpha \frac { \alpha } { ( q - p \alpha ) ^ { 4 } } \int _ { \alpha } ^ { 1 } d x _ { 2 } \int _ { 0 } ^ { 1 - x _ { 2 } } d x _ { 1 } \left[( 1 - t ) \left[2 A_{1}-2 A_{2}-A_{3}+3 A_{4}+2 V_{1}+2 V_{2}-4 V_{3}\right.\right.\right. \\
& \left.\left.+2 V_{4}-4 V_{5}\right]+(1+t)\left[2 P_{1}-2 P_{2}+2 S_{1}-2 S_{2}+2 T_{2}+4 T_{3}-6 T_{5}-4 T_{7}\right]\right]\left(x_{1}, x_{2}, 1-x_{1}-x_{2}\right) \\
& +2 \int_{0}^{1} d \alpha \frac{\alpha}{(q-p \alpha)^{4}} \int_{\alpha}^{1} d x_{3} \int_{0}^{1-x_{3}} d x_{1}\left[(1+t)\left[-P_{1}+P_{2}-S_{1}+S_{2}-T_{1}+T_{5}+T_{7}+T_{8}\right]\right] \\
& \times\left(x_{1}, 1-x_{1}-x_{3}, x_{3}\right)
\end{aligned}
$$




$$
\begin{aligned}
& +2 \int_{0}^{1} d \beta \int_{\beta}^{1} d \alpha \frac{1}{(q-p \beta)^{4}} \int_{\alpha}^{1} d x_{2} \int_{0}^{1-x_{2}} d x_{1}\left[(1+t)\left[-T_{2}+T_{3}+T_{4}-T_{5}-T_{7}-T_{8}\right]\right] \\
& \times\left(x_{1}, x_{2}, 1-x_{1}-x_{2}\right) \\
& -2 \int_{0}^{1} d \beta \int_{\beta}^{1} d \alpha \frac{1}{(q-p \beta)^{4}} \int_{\alpha}^{1} d x_{3} \int_{0}^{1-x_{3}} d x_{1}\left[(1+t)\left[T_{2}-T_{3}-T_{4}+T_{5}+T_{7}+T_{8}\right]\right] \\
& \times\left(x_{1}, 1-x_{1}-x_{3}, x_{3}\right) \\
& +8 m_{N(1535)}^{2} \int_{0}^{1} d \beta \int_{\beta}^{1} d \alpha \frac{\beta^{2}}{(q-p \beta)^{6}} \int_{\alpha}^{1} d x_{2} \int_{0}^{1-x_{2}} d x_{1}\left[(1+t)\left[-T_{2}+T_{3}+T_{4}-T_{5}-T_{7}-T_{8}\right]\right] \\
& \times\left(x_{1}, x_{2}, 1-x_{1}-x_{2}\right) \\
& -8 m_{N(1535)}^{2} \int_{0}^{1} d \beta \int_{\beta}^{1} d \alpha \frac{\beta^{2}}{(q-p \beta)^{6}} \int_{\alpha}^{1} d x_{3} \int_{0}^{1-x_{3}} d x_{1}\left[(1+t)\left[T_{2}-T_{3}-T_{4}+T_{5}+T_{7}+T_{8}\right]\right] \\
& \left.\times\left(x_{1}, 1-x_{1}-x_{3}, x_{3}\right)\right\},
\end{aligned}
$$

where $A_{i}, P_{i}, V_{i}, S_{i}$, and $T_{i}$ are distribution amplitudes of different twists. They have been expressed with respect to $N(1535)$ state wave functions. The explicit forms of these wave functions are presented in Ref. [40]. To eliminate contributions coming from the excited and continuum states, the Borel transformation and continuum subtraction are performed. The suppression of the excited and continuum states can be accomplished by means of the subsequent subtraction rules [41]:

$$
\begin{aligned}
\int d z \frac{\rho(z)}{(q-z p)^{2}} & \rightarrow-\int_{x_{0}}^{1} \frac{d z}{z} \rho(z) e^{-s(z) / M^{2}} \\
\int d z \frac{\rho(z)}{(q-z p)^{4}} & \rightarrow \frac{1}{M^{2}} \int_{x_{0}}^{1} \frac{d z}{z^{2}} \rho(z) e^{-s(z) / M^{2}}+\frac{\rho\left(x_{0}\right)}{Q^{2}+x_{0}^{2} m_{N}^{2}} e^{-s_{0} / M^{2}}, \\
\int d z \frac{\rho(z)}{(q-z p)^{6}} & \rightarrow-\frac{1}{2 M^{4}} \int_{x_{0}}^{1} \frac{d z}{z^{3}} \rho(z) e^{-s(z) / M^{2}}-\frac{1}{2 M^{2}} \frac{\rho\left(x_{0}\right)}{x_{0}\left(Q^{2}+x_{0}^{2} m_{N}^{2}\right)} e^{-s_{0} / M^{2}} \\
& +\frac{1}{2} \frac{x_{0}^{2} e^{-s_{0} / M^{2}}}{Q^{2}+x_{0}^{2} m_{N}^{2}}\left[\frac{d}{d x_{0}} \frac{\rho\left(x_{0}\right)}{x_{0}\left(Q^{2}+x_{0}^{2} m_{N}^{2}\right)}\right],
\end{aligned}
$$

where

$$
\begin{aligned}
s(z) & =(1-z) m_{N}^{2}+\frac{1-z}{z} Q^{2}, \\
x_{0} & =\frac{\sqrt{\left(Q^{2}+s_{0}-m_{N}^{2}\right)^{2}+4 m_{N}^{2} Q^{2}}-\left(Q^{2}+s_{0}-m_{N}^{2}\right)}{2 m_{N}^{2}} .
\end{aligned}
$$

The residue of the nucleon, $\lambda_{N}$, is needed for the numerical computation of $N(1535) \rightarrow N$ transition tensor form factors. The $\lambda_{N}$ is specified from two-point QCD sum rules [19]:

$$
\lambda_{N}=\left[e^{m_{N}^{2} / M^{2}}\left\{\frac{M^{6}}{256 \pi^{4}}\left(5+2 t+t^{2}\right) E_{2}(y)-\frac{\langle\bar{q} q\rangle^{2}}{6}\left(6\left(1-t^{2}\right)-(1-t)^{2}-\frac{m_{0}^{2}}{4 M^{2}}\left[12\left(1-t^{2}\right)-(1-t)^{2}\right]\right)\right]^{1 / 2},\right.
$$

where

$$
y=s_{0} / M^{2}
$$

and

$$
E_{n}(y)=1-e^{-y} \sum_{i=0}^{n} \frac{y^{i}}{i !}
$$


TABLE I. Input parameters of the $N(1535)$ state DAs for the two different sets.

\begin{tabular}{lcccccccrr}
\hline \hline Model & $\left|\lambda_{1}^{N(1535)} / \lambda_{1}^{N}\right|$ & $f_{N(1535)} / \lambda_{1}^{N(1535)}$ & $\varphi_{10}$ & $\varphi_{11}$ & $\varphi_{20}$ & $\varphi_{21}$ & $\varphi_{22}$ & $\eta_{10}$ & $\eta_{11}$ \\
\hline LCSR-I & 0.633 & 0.027 & 0.36 & -0.95 & 0 & 0 & 0 & 0 & 0.94 \\
LCSR-II & 0.633 & 0.027 & 0.37 & -0.96 & 0 & 0 & 0 & -0.29 & 0.23 \\
\hline \hline
\end{tabular}

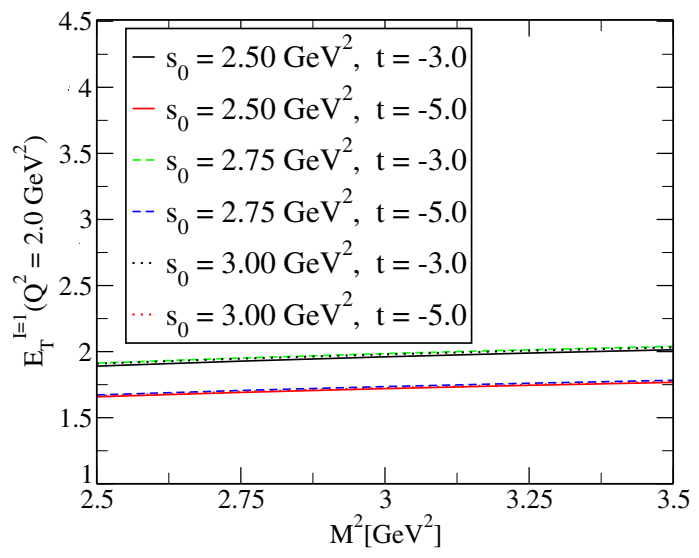

(a)

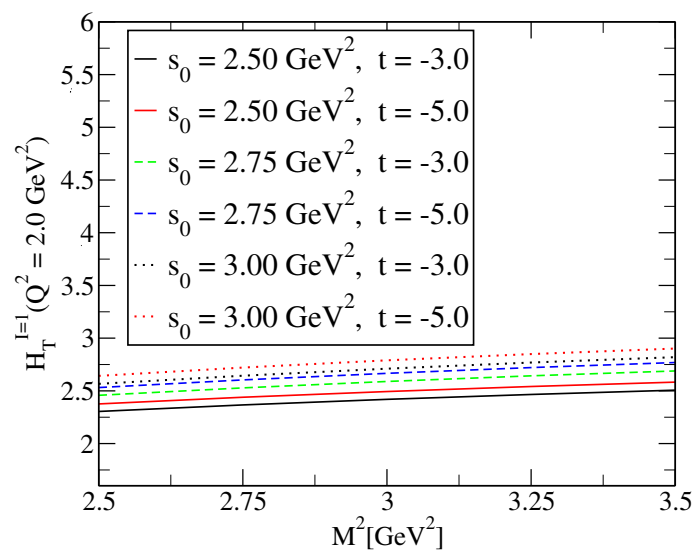

(c)

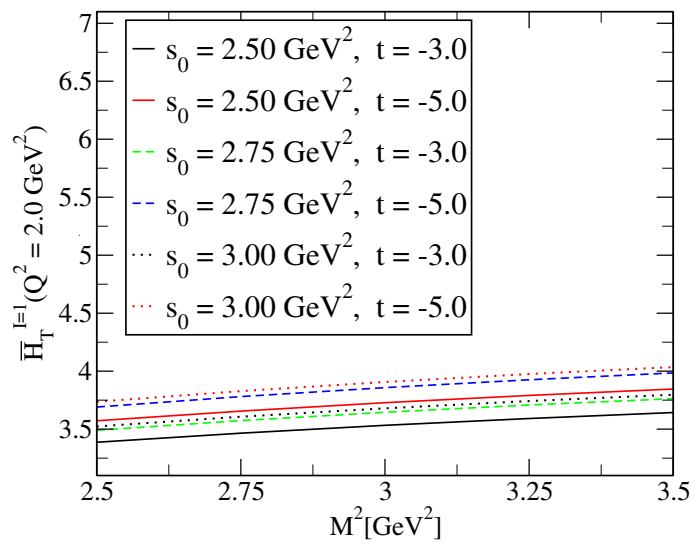

(e)

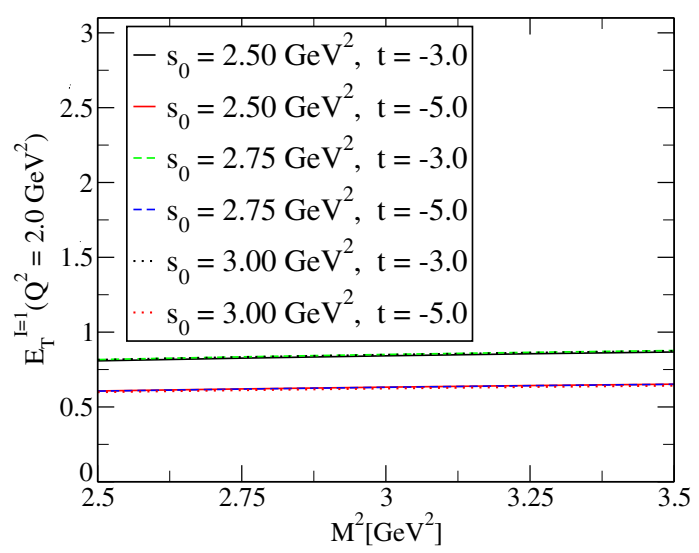

(b)

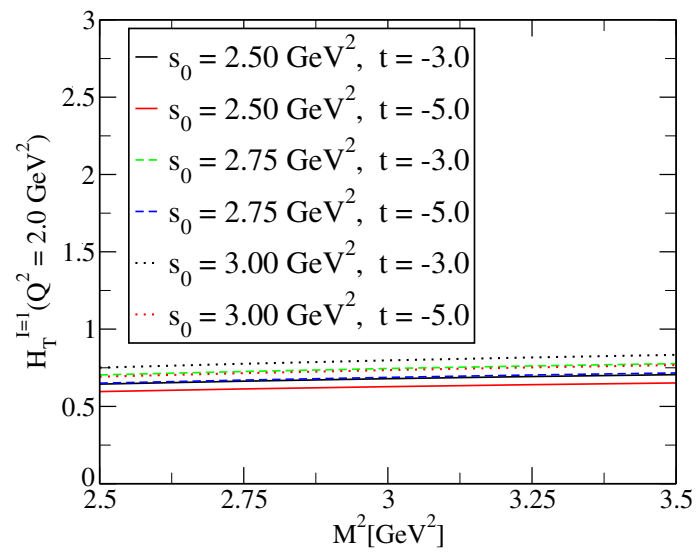

(d)

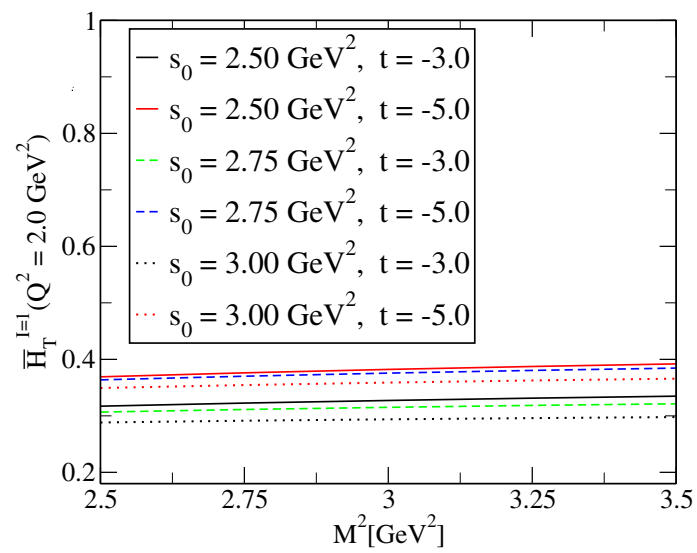

(f)

FIG. 1. The dependence of the isovector tensor form factors of the $N(1535) \rightarrow N$ transition on $M^{2}$ at $Q^{2}=2.0 \mathrm{GeV}^{2}$ and different values of $s_{0}$ and $t$ at their working windows. (a),(c),(e) for LCSR-I and (b),(d),(f) for LCSR-II. 


\section{NUMERICAL ANALYSIS AND CONCLUSION}

In this section, we have obtained the numerical analysis of $N(1535) \rightarrow N$ transition tensor form factors. The DAs of $N(1535)$ state have been evaluated by means of the light-cone QCD sum rule in Ref. [40]. The numerical values of the input parameters inside the DAs of the $N(1535)$ state are given in Table I, which are obtained at renormalization scale $\mu^{2}=$ $2.0 \mathrm{GeV}^{2}$. Furthermore, we use $\lambda_{1}^{N} m_{N}=-3.88(2)(19) \times$ $10^{-2} \mathrm{GeV}^{3}$ and $\lambda_{2}^{N(1535)} m_{N(1535)}=8.97(45) \times 10^{-2} \mathrm{GeV}^{3}$, given in Ref. [42] at renormalization $\mu^{2}=4.0 \mathrm{GeV}^{2}$, by rescaling to $\mu^{2}=2.0 \mathrm{GeV}^{2}$. Beside these values, we use $m_{N(1535)}=1.51 \pm 0.01 \mathrm{GeV}, m_{N}=0.94 \mathrm{GeV}$ [43], $m_{0}^{2}=$ $0.8 \pm 0.1 \mathrm{GeV}^{2}$, and $\langle\bar{q} q\rangle=(-0.24 \pm 0.01)^{3} \mathrm{GeV}^{3}$ [44]

The predictions for the isovector and isoscalar tensor form factors depend on three auxiliary parameters: the Borel mass parameter $M^{2}$, arbitrary mixing parameter $t$, and continuum threshold $s_{0}$. For the quality of the numerical values of the physical observables, we should minimize the dependence of the results on these parameters. The $M^{2}$ can change in the interval that the results relatively weakly depend on it with respect to the standard definition. The upper limit of it is acquired by demanding the maximum pole contributions, and its lower limit is acquired from the convergence of the operator product expansion and exceeding of the perturbative part over nonperturbative contributions. The $t$ is chosen such that the estimations of the isovector and isoscalar tensor form factors are reasonably insensitive of the values of $t$. The working region for the $s_{0}$ is chosen such that the maximum pole contribution is obtained, and the results relatively weakly depend on its choices. These constraints lead to the working intervals for auxiliary parameters as

$$
\begin{aligned}
2.50 \mathrm{GeV}^{2} & \leq M^{2} \leq 3.50 \mathrm{GeV}^{2}, \\
2.50 \mathrm{GeV}^{2} & \leq s_{0} \leq 3.00 \mathrm{GeV}^{2}, \\
-3.00 & \leq t \leq-5.00
\end{aligned}
$$

In Figs. 1 and 2, we show dependency of isovector and isoscalar tensor form factors with respect to the Borel mass parameter at three fixed values of the continuum threshold and two fixed values of the arbitrary mixing parameter in their working interval. The results show good stability

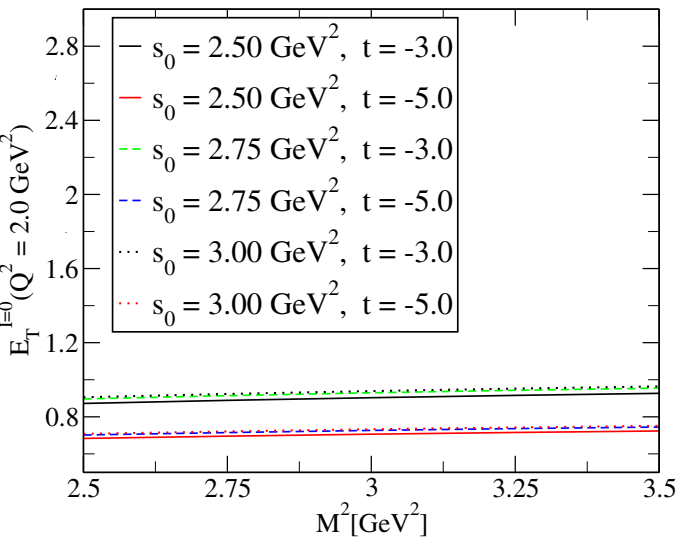

(b)

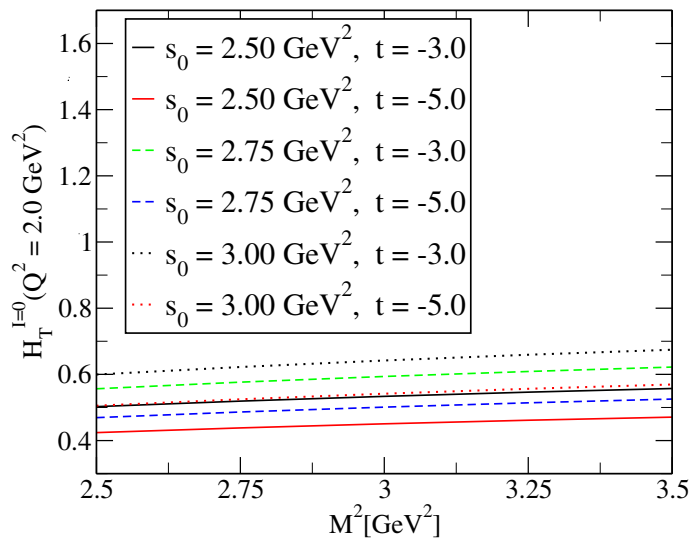

(d)

FIG. 2. The dependence of the isoscalar tensor form factors of the $N(1535) \rightarrow N$ transition on $M^{2}$ at $Q^{2}=2.0 \mathrm{GeV}^{2}$ and different values of $s_{0}$ and $t$ at their working windows. (a),(c) for LCSR-I and (b),(d) for LCSR-II. 


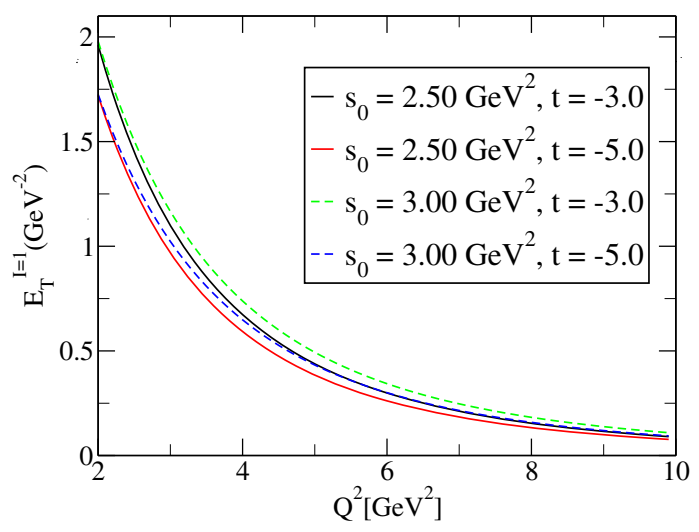

(a)

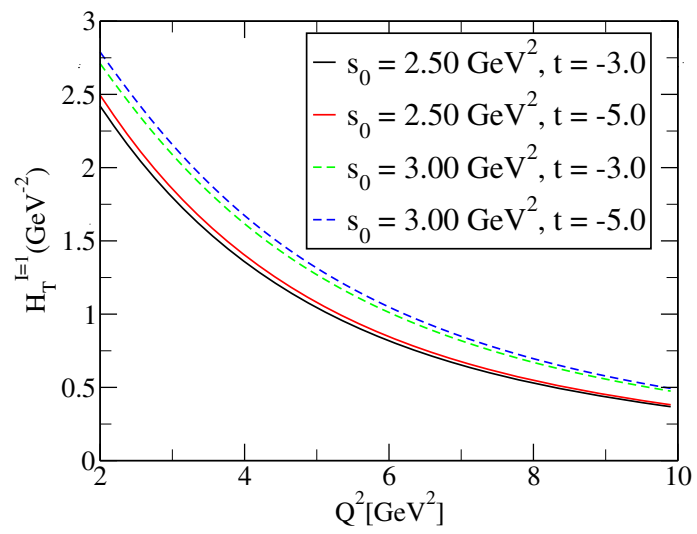

(c)

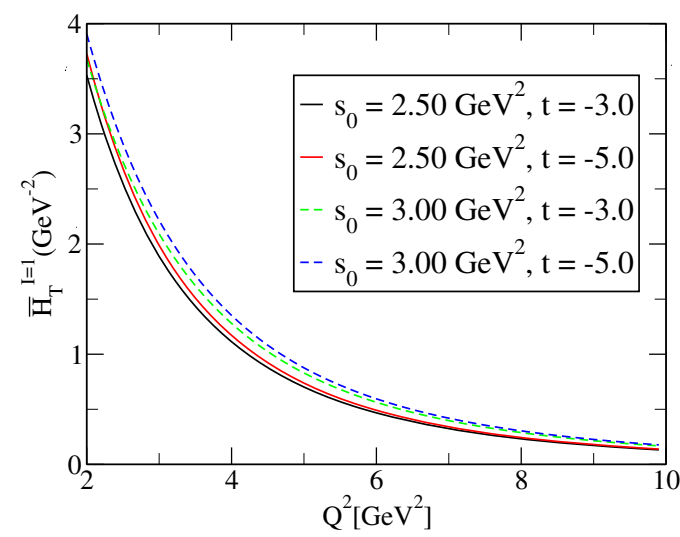

(e)

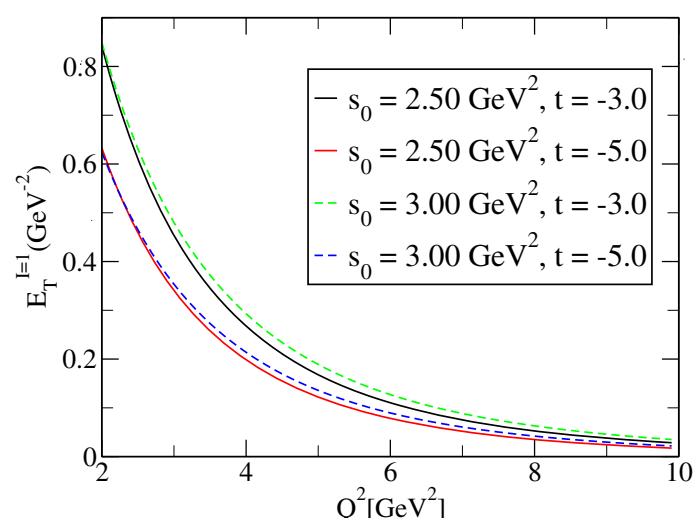

(b)

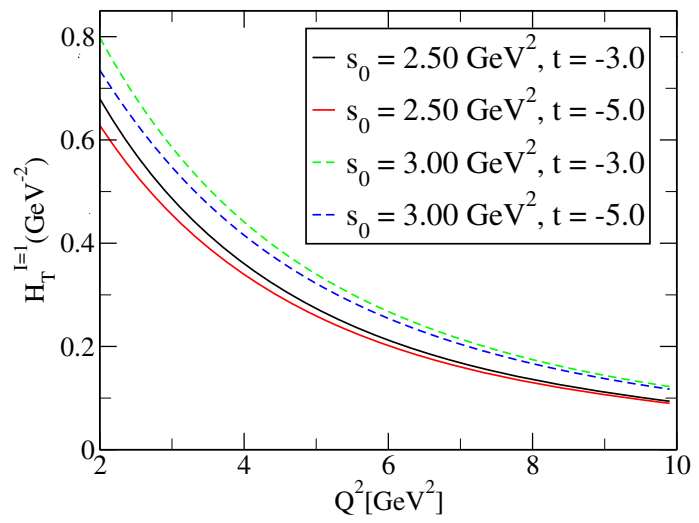

(d)

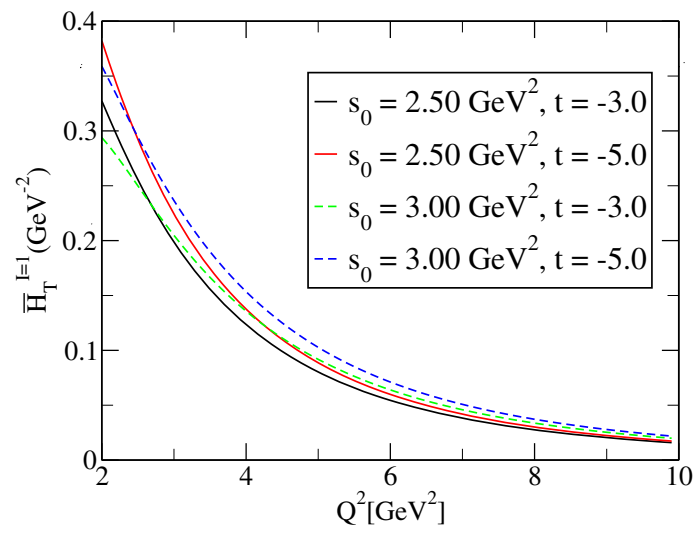

(f)

FIG. 3. The dependence of the isovector tensor form factors of $N(1535) \rightarrow N$ transition on $Q^{2}$ at $M^{2}=3.00 \mathrm{GeV}^{2}$ and different values of $s_{0}$ and $t$ at their working windows. (a),(c),(e) for LCSR-I and (b),(d),(f) for LCSR-II.

against the variations of the Borel mass parameters, as desired. In Figs. 3 and 4, we plot the dependence of the isovector and isoscalar tensor form factors on $Q^{2}$ for various values of $s_{0}$ and $t$ in their working regions and at the fixed values of $M^{2}=3.00 \mathrm{GeV}^{2}$ for LCSR-I and LCSR-II values of input parameters entering the DAs. All the form factors taken into account show a similar dependence on $Q^{2}$ for LCSR-I and LCSR-II except the form factor $\tilde{H}_{T}^{I=0}\left(Q^{2}\right)$. This form factor changes its sign in the region under consideration, so its results are not given in the text. We should note here that the light-cone QCD sum rule approach is trustworthy only for $Q^{2}>1.0 \mathrm{GeV}^{2}$. On the other hand, the baryon mass corrections of the DAs $\sim m^{2} / Q^{2}$ become very large for $Q^{2}<2.0 \mathrm{GeV}^{2}$; in other words, the light-cone QCD sum rules turn out to be untrustworthy. Thus, we expect the light-cone QCD sum rule to be more reliable and effective in the region of $2.0 \mathrm{GeV}^{2} \leq Q^{2} \leq 10.0 \mathrm{GeV}^{2}$. 


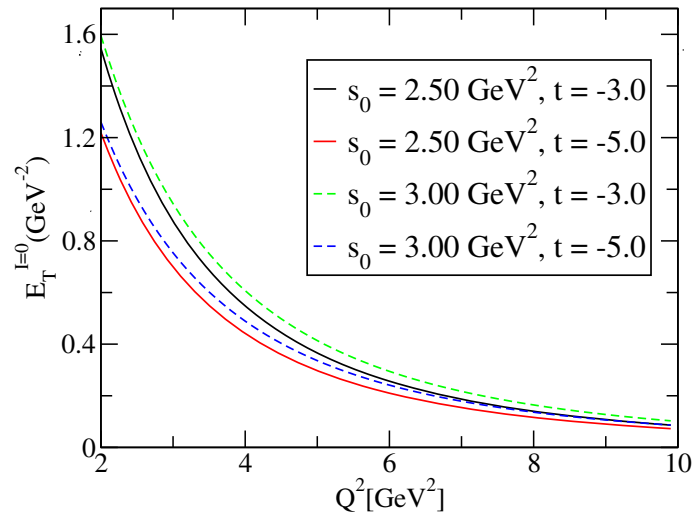

(a)

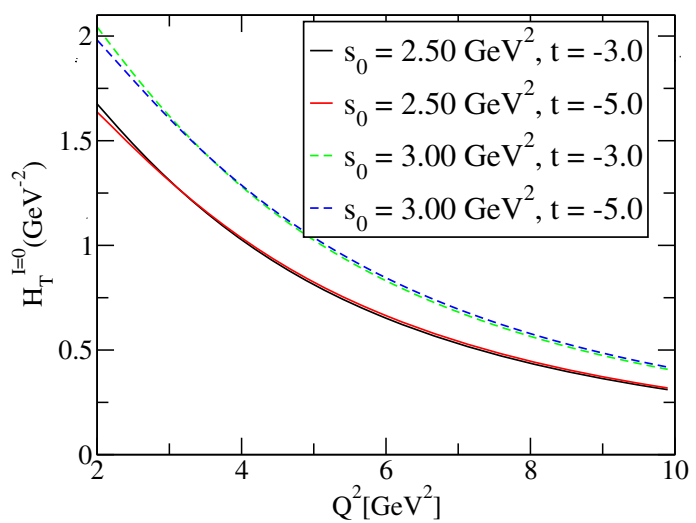

(c)

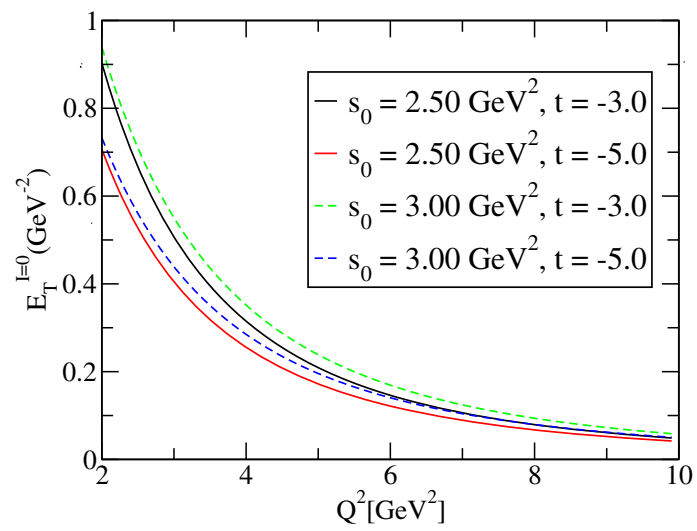

(b)

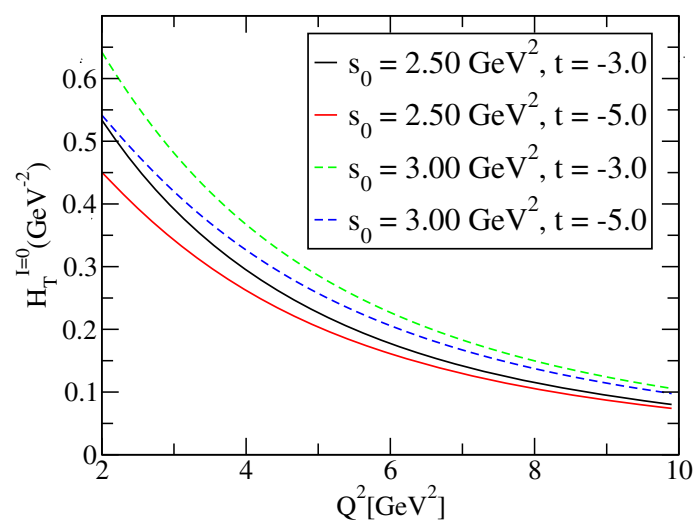

(d)

FIG. 4. The dependence of the isoscalar tensor form factors of $N(1535) \rightarrow N$ transition on $Q^{2}$ at $M^{2}=3.00 \mathrm{GeV}^{2}$ and different values of $s_{0}$ and $t$ at their working windows. (a),(c) for LCSR-I and (b),(d) for LCSR-II.

As we mentioned above, our sum rules work only for $Q^{2} \geq 2.0 \mathrm{GeV}^{2}$. However, we want to extend our analysis to the region $0 \leq Q^{2}<2$. To do this, some fit parameters need to be used. Our numerical investigations indicate that the isovector and isoscalar tensor form factors of $N(1535) \rightarrow N$ transition are nicely defined by employing the $p$-pole fit function:

$$
\mathcal{F}\left(Q^{2}\right)=\frac{\mathcal{F}(0)}{\left(1+Q^{2} /\left(p m_{p}^{2}\right)\right)^{p}}
$$

The numerical results obtained for $N(1535) \rightarrow N$ transition isovector and isoscalar tensor form factors are given in Table II. The results obtained by employing LCSR-I and LCSR-II parameters were found to be quite different from each other. The numerical values of the form factors $E_{T}^{I=0,1}$ $\left(Q^{2}=0\right), H_{T}^{I=0,1}\left(Q^{2}=0\right)$, and $\tilde{H}_{T}^{I=0,1}\left(Q^{2}=0\right)$ for the LCSR-II numerical values are smaller than those for the LCSR-I parameters. As one can see from Table I, the essential difference between input parameters of the DAs is the numerical values for the $\eta_{10}$ and $\eta_{11}$, which are related to the $p$-wave three-quark wave functions of the $N(1535)$ state

TABLE II. The obtained numerical values for the parameters of the isovector and isoscalar tensor form factors by employing the $p$-pole fit functions.

\begin{tabular}{|c|c|c|c|c|c|c|}
\hline \multirow[b]{2}{*}{ Form factors } & \multicolumn{3}{|c|}{ LCSR-I } & \multicolumn{3}{|c|}{ LCSR-II } \\
\hline & $\mathcal{F}(0)$ & $m_{p}(\mathrm{GeV})$ & $p$ & $\mathcal{F}(0)$ & $m_{p}(\mathrm{GeV})$ & $p$ \\
\hline$E_{T}^{I=1}\left(Q^{2}\right)$ & $7.54 \pm 1.26$ & $1.10 \pm 0.05$ & $3.6-4.0$ & $3.48 \pm 0.84$ & $1.07 \pm 0.07$ & $3.8-4.2$ \\
\hline$E_{T}^{I=0}\left(Q^{2}\right)$ & $5.05 \pm 1.01$ & $1.13 \pm 0.08$ & $3.6-4.0$ & $3.00 \pm 0.66$ & $1.14 \pm 0.10$ & $3.6-4.0$ \\
\hline$H_{T}^{I=1}\left(Q^{2}\right)$ & $5.22 \pm 0.27$ & $1.30 \pm 0.10$ & $3.0-3.4$ & $1.51 \pm 0.20$ & $1.28 \pm 0.10$ & $3.0-3.4$ \\
\hline$H_{T}^{I=0}\left(Q^{2}\right)$ & $3.37 \pm 0.47$ & $1.28 \pm 0.11$ & $3.0-3.4$ & $1.10 \pm 0.20$ & $1.32 \pm 0.10$ & $3.0-3.4$ \\
\hline $\bar{H}_{T}^{I=1}\left(Q^{2}\right)$ & $14.51 \pm 4.43$ & $1.02 \pm 0.10$ & $3.6-4.0$ & $1.30 \pm 0.39$ & $1.18 \pm 0.14$ & $3.6-4.0$ \\
\hline $\bar{H}_{T}^{I=0}\left(Q^{2}\right)$ & $\ldots$ & $\ldots$ & $\ldots$ & $\ldots$ & $\ldots$ & $\cdots$ \\
\hline
\end{tabular}


and, therefore, to the distribution of orbital angular momentum. This means these form factors are very sensitive to the shape parameters of the DAs of the $N(1535)$ state that parametrize relative orbital angular momentum of the quarks.

In summary, we have applied isovector and isoscalar tensor current to evaluate the tensor form factors of the $N(1535) \rightarrow N$ transition with the help of the light-cone QCD sum rule method. In numerical computations, we have used the most general forms of the interpolating current for the nucleon and the tensor current together with two different sets of the input parameters in the DAs of the $N(1535)$ state. We have obtained that the values of $N(1535) \rightarrow N$ transition tensor form factors are very sensitive to the input parameters of the DAs of the $N(1535)$ state. We have acquired that the $Q^{2}$ dependence of $N(1535) \rightarrow$ transition tensor form factors are well defined by a $p$-pole fit function. To our knowledge, this is the first study in the literature committed to the examination of the $N(1535) \rightarrow N$ transition tensor form factors. Thus, experimental data or theoretical predictions are not yet available to compare our numerical results with them. A comparison of the results acquired with the estimations of other theoretical approximations, such as the quark model, chiral perturbation theory, lattice QCD, etc., would also be interesting.

\section{ACKNOWLEDGMENTS}

The author is thankful to K. Azizi for helpful remarks, comments, and discussions.
[1] X.-D. Ji, Gauge-Invariant Decomposition of Nucleon Spin, Phys. Rev. Lett. 78, 610 (1997).

[2] A. V. Radyushkin, Nonforward parton distributions, Phys. Rev. D 56, 5524 (1997).

[3] P. Hoodbhoy and X.-D. Ji, Helicity flip off forward parton distributions of the nucleon, Phys. Rev. D 58, 054006 (1998).

[4] M. Diehl, Generalized parton distributions with helicity flip, Eur. Phys. J. C 19, 485 (2001).

[5] K. Goeke, M. V. Polyakov, and M. Vanderhaeghen, Hard exclusive reactions and the structure of hadrons, Prog. Part. Nucl. Phys. 47, 401 (2001).

[6] M. Anselmino, M. Boglione, U. D’Alesio, A. Kotzinian, F. Murgia, A. Prokudin, and C. Turk, Transversity and collins functions from SIDIS and e+ e- data, Phys. Rev. D 75, 054032 (2007).

[7] E. S. Ageev et al., A New measurement of the Collins and Sivers asymmetries on a transversely polarised deuteron target, Nucl. Phys. B765, 31 (2007).

[8] A. Airapetian et al., Single-Spin Asymmetries in SemiInclusive Deep-Inelastic Scattering on a Transversely Polarized Hydrogen Target, Phys. Rev. Lett. 94, 012002 (2005).

[9] K. Abe et al., Measurement of Azimuthal Asymmetries in Inclusive Production of Hadron Pairs in e+ e- Annihilation at Belle, Phys. Rev. Lett. 96, 232002 (2006).

[10] I. C. Cloet, W. Bentz, and A. W. Thomas, Transversity quark distributions in a covariant quark-diquark model, Phys. Lett. B 659, 214 (2008).

[11] H.-X. He and X.-D. Ji, The Nucleon's tensor charge, Phys. Rev. D 52, 2960 (1995).

[12] H.-X. He and X.-D. Ji, QCD sum rule calculation for the tensor charge of the nucleon, Phys. Rev. D 54, 6897 (1996).

[13] L. P. Gamberg and G. R. Goldstein, Flavor Spin Symmetry Estimate of the Nucleon Tensor Charge, Phys. Rev. Lett. 87, 242001 (2001).
[14] I. Schmidt and J. Soffer, Melosh rotation and the nucleon tensor charge, Phys. Lett. B 407, 331 (1997).

[15] B. Pasquini, M. Pincetti, and S. Boffi, Chiral-odd generalized parton distributions in constituent quark models, Phys. Rev. D 72, 094029 (2005).

[16] C. Lorce, Tensor charges of light baryons in the infinite momentum frame, Phys. Rev. D 79, 074027 (2009).

[17] T. Ledwig, A. Silva, and H.-C. Kim, Tensor charges and form factors of SU(3) baryons in the self-consistent $\mathrm{SU}(3)$ chiral quark-soliton model, Phys. Rev. D 82, 034022 (2010).

[18] G. Erkol and A. Ozpineci, Tensor form factors of nucleon in QCD, Phys. Lett. B 704, 551 (2011).

[19] T. M. Aliev, K. Azizi, and M. Savci, Nucleon tensor form factors induced by isovector and isoscalar currents in QCD, Phys. Rev. D 84, 076005 (2011).

[20] S. Pisano and M. Radici, Di-hadron fragmentation and mapping of the nucleon structure, Eur. Phys. J. A 52, 155 (2016).

[21] P. Hagler et al., Nucleon generalized parton distributions from full lattice QCD, Phys. Rev. D 77, 094502 (2008).

[22] M. Gockeler, P. Hagler, R. Horsley, D. Pleiter, P. E. L. Rakow, A. Schafer, G. Schierholz, and J. M. Zanotti, Quark helicity flip generalized parton distributions from two-flavor lattice QCD, Phys. Lett. B 627, 113 (2005).

[23] T. Bhattacharya, V. Cirigliano, S. Cohen, R. Gupta, A. Joseph, H.-W. Lin, and B. Yoon, Iso-vector and Iso-scalar tensor charges of the nucleon from lattice QCD, Phys. Rev. D 92, 094511 (2015).

[24] T. Gutsche, M. A. Ivanov, J. G. Korner, S. Kovalenko, and V. E. Lyubovitskij, Nucleon tensor form factors in a relativistic confined quark model, Phys. Rev. D 94, 114030 (2016).

[25] J. M. Olness, The Nucleon 'tensor charges' and the Skyrme model, Phys. Rev. D 47, 2136 (1993).

[26] A. kucukarslan, U. Ozdem, and A. Ozpineci, Tensor form factors of the octet hyperons in QCD, Phys. Rev. D 94, 094010 (2016). 
[27] I. Bedlinskiy et al., Measurement of Exclusive $\pi^{0}$ Electroproduction Structure Functions and their Relationship to Transversity GPDs, Phys. Rev. Lett. 109, 112001 (2012).

[28] I. Bedlinskiy et al., Exclusive $\pi^{0}$ electroproduction at $W>2 \mathrm{GeV}$ with CLAS, Phys. Rev. C 90, 025205 (2014); 90, 039901(A) (2014).

[29] M. Defurne et al., Rosenbluth Separation of the $\pi^{0}$ Electroproduction Cross Section, Phys. Rev. Lett. 117, 262001 (2016).

[30] I. Bedlinskiy et al., Exclusive $\eta$ electroproduction at $W>2 \mathrm{GeV}$ with CLAS and transversity generalized parton distributions, Phys. Rev. C 95, 035202 (2017).

[31] I. Aznauryan et al., Studies of nucleon resonance structure in exclusive meson electroproduction, Int. J. Mod. Phys. E 22, 1330015 (2013).

[32] V. M. Braun and I. E. Filyanov, QCD sum rules in exclusive kinematics and pion wave function, Yad. Fiz. 50, 818 (1989) [Z. Phys. C 44, 157 (1989)].

[33] I. I. Balitsky, V. M. Braun, and A. V. Kolesnichenko, Radiative decay $\sigma^{+} \rightarrow p \gamma$ in quantum chromodynamics, Nucl. Phys. B312, 509 (1989).

[34] V.L. Chernyak and I. R. Zhitnitsky, $B$ meson exclusive decays into baryons, Nucl. Phys. B345, 137 (1990).

[35] I. V. Anikin, V. M. Braun, and N. Offen, Electroproduction of the $N^{*}(1535)$ nucleon resonance in QCD, Phys. Rev. D 92, 014018 (2015).
[36] T. M. Aliev, T. Barakat, and K. Simsek, $N^{*}(1535) \rightarrow N$ transition form-factors due to the axial current, Phys. Rev. D 100, 054030 (2019).

[37] U. Özdem and K. Azizi, Gravitational transition form factors of $N(1535) \rightarrow N$, Phys. Rev. D 101, 054031 (2020).

[38] P. Hagler, Hadron structure from lattice quantum chromodynamics, Phys. Rep. 490, 49 (2010).

[39] M. Göckeler, P. Hägler, R. Horsley, Y. Nakamura, D. Pleiter, P. E. L. Rakow, A. Schäfer, G. Schierholz, H. Stüben, and J. M. Zanotti, Transverse Spin Structure of the Nucleon from Lattice QCD Simulations, Phys. Rev. Lett. 98, 222001 (2007).

[40] M. Emmerich, Decay Form factors for $\Lambda_{b, c}$ and B with QCD sum rules, Ph.D. Thesis, Regensburg University, 2018.

[41] V. M. Braun, A. Lenz, and M. Wittmann, Nucleon form factors in QCD, Phys. Rev. D 73, 094019 (2006).

[42] V. M. Braun, S. Collins, B. Gläßle, M. Göckeler, A. Schäfer, R. W. Schiel, W. Söldner, A. Sternbeck, and P. Wein, Lightcone distribution amplitudes of the nucleon and negative parity nucleon resonances from lattice QCD, Phys. Rev. D 89, 094511 (2014).

[43] M. Tanabashi et al., Review of particle physics, Phys. Rev. D 98, 030001 (2018).

[44] B. L. Ioffe, QCD at low energies, Prog. Part. Nucl. Phys. 56, 232 (2006). 